\title{
Modelización de problemas para desarrollar habilidades de experimentación
}

Recibido: 05-11-2010| Aceptado: 30-06-2011

\author{
Modeling problems in developing experimental skills
}

José Joaquín García García*

Edilma Rentería Rodríguez**

Resumen: Este artículo presenta los resultados de la aplicación de una estrategia didáctica que propone la resolución de problemas prácticos contextualizados, usando la modelización, para mejorar las habilidades de experimentación de los estudiantes de undécimo grado. Esta investigación tuvo un enfoque cuasi experimental con un grupo experimental, un grupo control y una prueba posterior aplicada simultáneamente a ambos grupos. Los resultados muestran como el grupo experimental desarrolló mucho más las habilidades de experimentación que el grupo control. Las diferencias entre los grupos, pueden deberse a que en la estrategia didáctica tradicional usada en el grupo control la resolución de problemas es sólo marginal y cuando se propone es para resolver problemas cerrados usando series de algoritmos, lo que no requiere del uso de conceptos como relación causal o hipótesis, además en ésta, las actividades experimentales también son marginales y sólo son usadas como posibilidades de aplicación y comprobación del conocimiento, excluyendo características verdaderas de los experimentos como el control y la manipulación de variables.

Palabras clave: Resolución de problemas, modelización, habilidades de experimentación

\begin{abstract}
This article presents the results of implementing a teaching strategy that proposes the solution of practical problems in context, using modeling to improve the experimentation skills of eleventh graders. This research study used a quasi-experimental approach with an experimental group, a control group and a post test applied simultaneously to both groups. The results show that the experimental group developed more experimentation skills than the control group. Differences between groups may be due to the traditional teaching strategy used in the control group problem solving is only marginal as is proposed to solve closed problems by using series of algorithms, which does not require the use of concepts like causal relationship or hypothesis, besides the experimental activities, are also marginal and are only used as possible applications and verification of knowledge, excluding real characteristics of the experiments as the control and manipulation of variables.
\end{abstract}

Keywords: Problem solving, modeling, experimentation skills

* Docente titular Facultad de Educación. Universidad de Antioquia. yocolombiano@yahoo.com.mx

** Docente catedrática Facultad de Educación. Universidad de Antioquia. renteriaedilma@hotmail.com 


\section{Introducción}

En la educación en ciencias puede enseñarse los conocimientos científicos como tales, sus fundamentos epistemológicos, históricos y sociológicos, los procedimientos utilizados para construir dichos conocimientos y acerca del uso socialmente responsable de estos conocimientos científicos. De esta forma es menester de los docentes de ciencias el usar y probar estrategias que traten de responder al máximo a estas exigencias múltiples en el campo de la educación en ciencias. Es por ello, que en este trabajo se diseña y prueba una estrategia que propone la resolución de problemas abiertos y contextualizados usando procedimientos de modelización experimental, como una estrategia que integra la enseñanza de los conceptos y las teorías científicas, con la de los procedimientos de su producción y el contexto de uso de las ciencias, en un marco epistemológico que propone a la ciencia como una actividad y como una construcción de las sociedades humanas. Este trabajo se centra sólo en el análisis del efecto de la estrategia didáctica alternativa propuesta en el desarrollo de determinadas habilidades de experimentación propias del campo llamado del saber hacer la ciencia. Este interés obedece a que la necesidad de determinar si es posible mejorar dichas habilidades y con ello contribuir al empoderamiento de las sociedades en su capacidad de producir conocimientos científicos. La investigación aquí propuesta usa un método cuasi experimental con grupos control y experimental y la aplicación de una pos prueba acerca de las habilidades de experimentación. La estrategia didáctica propuesta y probada aquí se enmarca en las propuestas de ciencia integradora de Derek Hodson (2003), que propone que se debe enseñar ciencias, sobre la ciencia, a hacer ciencia y a usar responsablemente la ciencia; en las propuestas epistemológi- cas de Gerard Fourez (1994) que ponen a la modelización en el centro de la producción de conocimientos científicos y, en los trabajos referidos al uso de la modelización en las aulas de clase realizados por varios autores Halloun, 1996; Martinand, 1986; Crawford y Cullin, 2004; Justi, 2002).

\section{La naturaleza de los modelos y la modelización}

Un modelo es una estructura o construcción hipotética deductiva, probable y heurística de carácter idealizado, sobre realidades inaccesibles directamente. En un modelo se representan correlaciones entre los fenómenos a través de una representación conceptual esquemática (Martinand, 1986; Guidoni, 1989; Jiménez y Perales, 2002). Por otra parte, un modelo es un instrumento de investigación en las ciencias para la formulación y prueba de hipótesis, a través de experimentos, además de ser utilizado para la descripción y comunicación de los fenómenos (Black, 1962; Van Driel y Verloop, 1999). A su vez, los modelos son el resultado de expresar dichas hipótesis, por lo tanto a partir de ellos se pueden generar predicciones (Crawford y Cullin, 2004).

Es importante decir que los modelos sólo se concentran en aspectos específicos de los sistemas para describirlos, explicarlos y predecir su comportamiento (Ingham y Gilbert, 1991; Raviolo, Martínez y Aznar, 2001). Así, en la ciencia al construir los modelos se hace un corte en la realidad considerando sólo lo importante, abstrayéndolo, simplificándolo y elaborando analogías sobre ello (Galagovsky y Adúriz - Bravo, 2001).

Igualmente, la modelización es un fenómeno cíclico a través del cual se pueden producir o no predicciones a partir del modelo, además de generarse constan- 
tes replanteamientos y modificaciones del mismo (Fourez, 1994; Rubinstein, 1996). Así, el proceso de modelización no se termina en el análisis de los resultados sino que es continuo y por ello incluye de forma sucesiva validaciones, análisis y construcción de modelos intermedios que se refinan progresivamente (García, 2011)

Autores como Halloun, (1996); Hestenes, (1995) y Estanya (2001) proponen que en la enseñanza de las ciencias se debe se utilizar los modelos con el objetivo de describir, explicar y predecir situaciones, en el marco de investigaciones sobre causa-efecto y que para ello, se deben elegir los factores relevantes del sistema a estudiar y declarar las hipótesis sobre sus relaciones. Para probar dichas hipótesis se debe realizar un trabajo experimental que incluye obtener resultados, diferenciarlos y compararlos, reflexionando sobre ellos para construir y escribir interpretaciones adecuadas. Finalmente, este mismo grupo de autores propone que el modelo construido y probado debe ser ampliado a otros sistemas y extrapolado a la construcción de otros modelos.

\section{La relación entre la modelización y la experimentación}

Todo experimento corresponde a un modelo esquemático de sucesos que tiene como fin construir solo algunos aspectos de un fenómeno. De esta manera, un experimento es un acontecimiento reconstruido imitando la realidad. Así, en un experimento los montajes específicos, la lectura de resultados y la interpretación de datos, siempre implican la construcción y utilización de modelos abstractos (Arcà y Guidoni, 1989). Por otra parte, la experimentación posibilita un proceso de construcción de elementos para la interpretación de los resultados experi- mentales, la construcción de explicaciones y su comunicación a la comunidad científica, es decir la construcción de modelos teóricos conceptuales explicativos sobre el comportamiento de los fenómenos (Gilbert, 1993, Tomasi, 1999; Justi, 2002). Además, a través de la experimentación de las hipótesis provenientes de los modelos, dichos modelos son puestos a prueba y contrastados, llevando a cabo actividades como identificación de variables, establecimiento de relaciones entre ellas, y elaboración de descripciones cualitativas o cuantitativas de los objetos (Jackson, 1995).

De acuerdo con lo anterior, como el modelizar experimentos requiere de la selección de variables desde un fenómeno y de sólo determinadas relaciones entre dichas variables, este procedimiento podría desarrollar habilidades de experimentación como la determinación de las características generales de las variables incluidas en el experimento modelado, y la identificación de las mismas como cualitativas o cuantitativa, dependientes o independientes, además del establecimiento de correlaciones entre ellas. Así mismo, la experiencia de modelizar experimentos que posibilita la construcción de modelos explicativos, puede desarrollar habilidades de experimentación referidas al manejo de la naturaleza de las relaciones entre las variables estudiadas, de su carácter causal y de cómo se articulan en la formulación de hipótesis, además de la habilidad para determinar las condiciones de confiabilidad de un experimento.

\section{Metodología}

Para el presente trabajo se usó como modelo investigativo el cuasi experimental con un grupo experimental, y otro control y una pos prueba diligenciada por ambos grupos. 
La muestra estuvo representada por dos grupos de estudiantes de undécimo grado, pertenecientes ambos a una estratificación socioeconómica popular, de la zona metropolitana de la ciudad de MedeIlín, Colombia. Al grupo experimental se le aplicó durante siete semanas la estrategia didáctica basada en la solución de problemas prácticos, usando la modelización, experimental, mientras que con el grupo control se utilizó una estrategia convencional, basada en la transmisión expositiva de conceptos y en la asimilación memorística de los mismos, durante un periodo de tiempo similar. Ambos grupos pertenecían a la misma Institución educativa, el colegio San Luis Gonzaga, ubicado en el municipio de Copacabana, y en los dos grupos fue la misma docente la que aplicó tanto la estrategia didáctica alternativa como la estrategia didáctica tradicional. Los grupos experimental y control no fueron elegidos al azar pues se trataba de grupos cautivos ya conformados antes de iniciar el estudio.

\section{Instrumento para la recolección de datos}

Para la recolección de la información de ambos grupos se utilizó una prueba acerca de las habilidades de experimentación en la que se indagaba por el conocimiento sobre el uso y la naturaleza de las variables y sobre las características de los procedimientos de experimentación. Esta prueba estuvo constituida por ocho preguntas con un formato de opción múltiple, referidas cada una a un indicador de las dimensiones de la variable habilidades de experimentación. Dicha prueba, hace parte de un test más amplio que mide la capacidad global para modelizar situaciones. Los reactivos de dicha prueba se sometieron a revisiones informales por otros investigadores, centrándose en la posible correlación entre el reactivo y el indicador a evaluar, y en su redacción gramatical (validación externa por pares). Además, para su selección se tuvo en cuenta que su índice de dificultad fuese aproximadamente de 0,50 ( $50 \%$ de los examinados debe responder de manera correcta al reactivo), y que tuviesen un alto índice de discriminación, es decir una buena capacidad para que se discrimine entre buenos y malos desempeños con respecto al indicador evaluado (para consultar información técnica acerca de la forma de construcción de estos dos índices ver Hogan, 1994). Para calcular el índice de discriminación y el índice de dificultad de cada pregunta se realizó una prueba piloto con 286 estudiantes. Así, por cada indicador se seleccionó el reactivo con mayor índice de discriminación y cuyo índice de dificultad fuese próximo a 0.5 .

Por otra parte, los ítems también fueron seleccionados calculando el índice de homogeneidad de cada uno de los reactivos, es decir el nivel de coherencia de cada ítem con el resto de la prueba (validación interna). Además, para determinar la confiabilidad de la prueba como medida de su coherencia interna se aplicó el índice de correlación Alfa Crombach (a), que oscila entre 0 y 1, 0 significa nula confiabilidad y 1 representa un máximo de confiabilidad. El valor del a de Crombach encontrado fue de 0,71 , lo que constituye un valor aceptable para una prueba con un reducido número de preguntas (Ver anexo 1).

\section{Hipótesis de investigación}

El uso de una estrategia didáctica basada en la articulación de procedimientos de modelización experimental a la resolución de problemas prácticos abiertos y contextualizados influye positivamente en el desarrollo de las habilidades de experimentación de los estudiantes. 
Dentro de las variables a estudiar se tienen:

Variable manipulada: estrategia didáctica. Esta variable presenta dos valores, el primero es la estrategia didáctica tradicional basada en los procesos de transmisión expositiva y asimilación memorística usada con el grupo control. El otro valor es la estrategia de enseñanza alternativa que propone la resolución de problemas usando la modelización experimental, y que es usada con el grupo experimental. Es importante anotar que en ambos grupos la estructura conceptual enseñada fue la misma y se refirió a los conocimientos básicos de la termodinámica, en la que se incluían los conceptos de temperatura, calor, energía interna calor específico, equilibrio térmico, presión, además de la primera y la segunda ley de la termodinámica $y$, de la comprensión del comportamiento de la temperatura durante y después de los cambios de fase de la materia. Por razones de espacio los resultados encontrados en el aspecto conceptual tras la aplicación de la estrategia didáctica alternativa no serán presentados aquí, y serán materia de otro artículo.

En la estrategia didáctica tradicional basada en los procesos de transmisión expositiva y asimilación memorística el estudiante es más bien un alumno que recibe conocimiento y no un estudiante que estudia los fenómenos con el fin deconstruir conocimiento. Por ello la mayoría de las acciones en el aula las realiza el profesor, esta estrategia está basada en la presentación secuencial y estructurada de los contenidos usando métodos expositivos, contenidos que luego son evaluados de forma reproductiva. La secuencia prototípica de una clase basada en la estrategia tradicional de transmisión expositiva y recepción memorística incluye los siguientes pasos:
- Introducción: en este paso se procede a la contextualización de nuevo contenido, tratando de conectarlo con los conocimientos anteriores de los estudiantes, sus experiencias y motivaciones.

- Presentación de nuevos conceptos: en esta fase se introducen los nuevos conceptos en forma de definiciones, clasificaciones, unidades, ejemplos o representaciones.

- Establecimiento de la relación con otros conceptos previamente definidos: en esta fase se enmarcan en contextos teóricos dichos conceptos para constituir ecuaciones, leyes, o teorías.

- Aplicación: esta fase propone la resolución de problemas lápiz y papel de carácter cuantitativo para aplicar los conceptos aprendidos, esta resolución se hace a partir de ejercicios de muestra desarrollados y resueltos por el profesor con anterioridad. Además esta fase incluye la realización de trabajos prácticos incluidos en manuales para comprobar los conceptos, leyes y teorías estudiados en el aula de clase.

La estrategia didáctica alternativa que integra la resolución de problemas y modelización, plantea a los estudiantes para su resolución un grupo de problemas cualitativos contextualizados, y de carácter experimental, para que él al resolverlos proponga hipótesis y las pruebe a través de un modelo experimental, que le provee de datos para calcular valores constantes, para resolver el problema cuantitativamente (Ver anexo 2). Los elementos principales de la estrategia son los siguientes:

- Una fase inicial de aclaración de conceptos y de comprensión de los procesos de resolución de problemas y de modelización. 
- Una fase de ejecución en la cual los estudiantes usaban una cartilla para resolver los problemas propuestos en los que se incluían los siguientes pasos a desarrollar en la resolución:

» Presentación a los estudiantes de problemas contextualizados, abiertos y cualitativos. Estos problemas son situaciones cuya resolución requiere de procedimientos distintos a la reproducción y aplicación de algoritmos y ecuaciones, ya que implican al razonamiento autónomo y deliberativo de los estudiantes para reorganizar y buscar la información científica necesaria (García, 2003).

Análisis comprensivo del problema y de los hechos presentados en él, determinando aspectos conocidos y desconocidos relevantes para solucionarlo, representándolo verbal y esquemáticamente.

»Acotación del problema describiendo los parámetros para su solución.

Emisión de hipótesis fundamentadas como soluciones al problema.

Elaboración de un plan de resolución usando un modelo experimental de laboratorio, reformulando experimentalmente el problema. Esto requiere de procedimientos teóricos o prácticos como búsqueda de información, sobre nexos, entre las variables estudiadas, y sobre métodos y estrategias para obtener datos sobre dichos vínculos, para procesarlos y para contrastar las hipótesis, haciendo lo siguiente:

Definir qué información se desea obtener del modelo físico. Identi- ficar, las variables experimentales (dependientes e independientes) y extrañas que se deben incluir y que, pueden influir el modelo. Seleccionar una representación adecuada para relacionar las variables escogidas, y sus unidades de medida. Construir el modelo físico y experimentar de acuerdo a lo planeado para la consecución de datos que permitan demostrar las hipótesis.

» Modelización verbal y matemática de las relaciones encontradas entre las variables, llevando a cabo los siguientes pasos:

» Determinar el objetivo del modelo simbólico que usa los datos experimentales.

Elaborar un modelo gráfico que relacione los datos de las variables estudiadas en el problema. Expresar verbalmente las relaciones funcionales encontradas entre las variables. Elaborar enunciados y ecuaciones sobre como se relacionan las variables. Analizar e interpretar la respuesta al problema a la luz de las hipótesis planteadas.

- Una fase de retroalimentación en la cual se evaluaban los modelos construidos por los estudiantes y las fallas y los aciertos que se habían presentado durante los procesos de diseño de los modelos experimentales, experimentación como tal y diseño de modelos simbólico matemáticos.

Dentro de las variables observadas se tuvo:

Variable habilidades de experimentación. Para el estudio de esta variable se tuvieron en cuenta dos factores, el conocimiento 
acerca de la naturaleza de la variable y el conocimiento básico acerca del proceso de experimentación. Para el primer factor se elaboraron los siguientes indicadores: concepción general de variable (CV), concepto de Variable cuantitativa (VC), naturaleza de la variable independiente $(\mathrm{VI})$, naturaleza de la variable dependiente (VD) y correlación entre variables (C).

Para el factor correspondiente al conocimiento acerca de la experimentación se tuvieron en cuenta los siguientes indicadores: concepto de relación causal (RC), concepto de hipótesis $(\mathrm{CH})$ y características de un experimento (CE).

A continuación se describen cada uno de estos factores y sus indicadores.

Factor conocimiento sobre la naturaleza de las variables. El término variable ha sido difícil de definir, esto debido a que éste tiene diferentes significados de acuerdo con el contexto de la situación en la cual se encuentre la variable. De esta manera una variable puede representar una incógnita especifica, un número generalizado, o una relación funcional (Morales y Díaz, 2003). Como incógnita específica una variable representa un determinado número, y se caracteriza porque tiene un único valor. Como número general una variable puede asumir múltiples valores. Como una función, la variable se asume como parte de una relación sistemática entre dos conjuntos de valores, en los que al variar un valor también hay variación del otro. El factor naturaleza de la variable es evaluado mediante la utilización de 5 indicadores, ellos son: concepción general de variable (CV), concepto de variable cuantitativa (VC), naturaleza de la variable independiente (VD), naturaleza de la variable dependiente (VI), correlación entre variables (C). A continuación se describe la forma como se operacionaliza cada indicador para ser evaluado.
- Concepción general de variable (CV): para evaluar este indicador se presenta una ecuación en la cual hay algunas magnitudes variables y algunas magnitudes constantes, $y$, el estudiante debe determinar cuáles son las magnitudes que varían en dicha ecuación.

- Concepto de variable cuantitativa (VC): las variables cuantitativas son aquellas que se expresan mediante cifras numéricas. Para evaluar este indicador se presenta a los estudiantes algunos enunciados que incluyen variables y el debe escoger el que presente únicamente variables cuantitativas.

- Naturaleza de la variable independiente (VI): la variable independiente representa la causa, que ocurre primero en el tiempo. Además, es la variable que puede ser manipulada por el investigador. Para evaluar este indicador se presenta un gráfico en el cual se relacionan dos variables $y$, el evaluado debe indicar cual es la variable independiente.

- Naturaleza de la variable dependiente (VD): la variable dependiente es aquella en la cual se evidencian los cambios debidos a la variable independiente. Así, representa el efecto que se produce por la causa o variable independiente. La variable dependiente no es manipulada, más bien es la variable observada. Para evaluar este indicador se pide a los estudiantes seleccionar la afirmación que mejor define la naturaleza de la variable dependiente.

- Correlación entre variables (C): dos o más variables están correlacionadas cuando al variar una de ellas sistemáticamente varía la otra. Para evaluar este indicador el estudiante debe seleccionar desde un grupo de enunciados que establecen relaciones entre variables cualitativas, cual muestra una correlación. 
Factor conocimiento sobre el proceso de experimentación. La experimentación es un proceso de investigación empírico. Dicho proceso se caracteriza porque en éste se controlan las condiciones naturales del fenómeno estudiado, con la intención de analizar los posibles efectos que ejercen unas variables sobre otras. De esta manera, el proceso de experimentación requiere de varios aspectos: en primer lugar, el investigador debe manipular la variable independiente para producir el efecto en la variable dependiente. En segundo lugar, se debe evaluar el efecto de la variable independiente, mediante la observación de la variable dependiente. En tercer lugar, se debe controlar la influencia de las variables extrañas.

El factor experimentación es evaluado mediante la utilización de 3 indicadores, ellos se refieren al manejo de los siguientes conceptos: relación causal (RC), concepto de hipótesis $(\mathrm{CH})$, y conocimiento de las características de un experimento (CE).

- Relación Causal (RC): el principio de causalidad postula que todo efecto es precedido por una causa, es decir, todos los eventos que ocurren lo hacen porque son efecto de otros que se han generado anteriormente. Para evaluar las relaciones causales se formula una situación en la que se establece una relación causal entre dos fenómenos y el evaluado debe determinar cual de ellos es la causa.

- Hipótesis (CH): la hipótesis se define como una explicación provisional de los hechos que puede ser verdadera o falsa. Para evaluar este indicador se formula un reactivo en el cual se debe seleccionar la mejor definición del término hipótesis.
- Características de un experimento (CE): con la realización de un experimento se determina el comportamiento de la variable dependiente, ocasionado por la variable independiente, para establecer dicha relación se deben controlar variables extrañas que pueden influir en la variable dependiente. Para evaluar este indicador se propone seleccionar desde varias opciones la que indique correctamente cuando un experimento es confiable.

\section{Resultados de la aplicación experimental de la estrategia didáctica}

\section{Sobre la comparación global de las habilidades de experimentación}

La comparación de las medias referidas a la variable habilidad de experimentación muestra que el grupo experimental tuvo un desarrollo mayor en dichas habilidades que el grupo control (5,57 y 3,10 respectivamente). Para determinar si esta diferencia es estadísticamente significativa se aplicó una prueba $t$ de Student. Los resultados de esta prueba muestran que la diferencia es estadísticamente significativa ( $t=5,832$ y $p=0,000)$. Estos resultados permiten probar la hipótesis propuesta en este trabajo y concluir de esta manera que el uso de una estrategia didáctica basada en la articulación de procedimientos de modelización experimental a la resolución de problemas prácticos abiertos y contextualizados influye positivamente en el desarrollo de las habilidades de experimentación de los estudiantes (Ver tabla 1). 


\begin{tabular}{|c|c|c|c|c|c|c|c|c|}
\hline & \multicolumn{5}{|c|}{ Diferencias relacionadas } & \multirow[t]{3}{*}{$\mathrm{t}$} & \multirow[t]{3}{*}{ gl } & \multirow{3}{*}{$\begin{array}{l}\text { Sig. } \\
\text { (bila- } \\
\text { teral) }\end{array}$} \\
\hline & \multirow[t]{2}{*}{ Media } & \multirow[t]{2}{*}{$\begin{array}{c}\text { Desviación } \\
\text { típ. }\end{array}$} & \multirow{2}{*}{$\begin{array}{l}\text { Error } \\
\text { típ. } \\
\text { de la } \\
\text { media }\end{array}$} & \multicolumn{2}{|c|}{$\begin{array}{l}95 \% \text { Intervalo de } \\
\text { confianza para la } \\
\text { diferencia }\end{array}$} & & & \\
\hline & & & & Inferior & Superior & & & \\
\hline $\begin{array}{l}\text { Habiexperim } \\
\text { GrupExper }\end{array}$ & 5,577 & 1,31 & 0,248 & 1,59734 & 3,33123 & 5,832 & 27 & ,000 \\
\hline $\begin{array}{l}\text { Habiexperim } \\
\text { Grupcontrol }\end{array}$ & 3,107 & 1,66 & 0,314 & & & & & \\
\hline
\end{tabular}

Tabla 1. Calculo de t de Student para la variable habilidades de experimentación Grupo control VS grupo experimental

\section{Sobre la comprensión de la naturaleza de las variables}

\section{- Concepto de variable cuantitativa (VC).}

Los resultados indican que el $68 \%$ del grupo control tiene conocimiento de lo que es una variable cuantitativa, y que el $32 \%$ de dicho grupo no lo tiene (gráfico 1). Igualmente, muestran que el $75 \%$ del grupo experimental comprende el concepto de variable cuantitativa, pero que, el $25 \%$ de este mismo grupo no lo comprende (gráfico 2). Las pequeñas diferencias entre los dos grupos pueden originarse en el hecho de que ambos grupos manipularon variables cuantitativas, aunque resolviesen diferentes tipos de problemas, el grupo control resolviendo problemas numéricos de lápiz y papel y el grupo experimental al construir modelos formales para representar los resultados y las relaciones halladas en los procesos experimentales. Así mismo, este resultado puede atribuirse al uso frecuente de las variables de carácter cuantitativo más que, de las del tipo cualitativo, en las clases de ciencias y matemáticas, lo que hace que los estudiantes estén ciertamente familiarizados con la naturaleza de este tipo de variables.
- Concepción general de variable (CV)

Los resultados muestran que el $18 \%$ del grupo control comprende lo que es una variable, y que el $82 \%$ de dicho grupo no lo comprende (gráfico 1). También estos resultados muestran que el $50 \%$ del grupo experimental comprenden el concepto de variable y que el otro $50 \%$ no tiene dicha comprensión (gráfico 2).

Estas diferencias pueden deberse a que mientras al grupo control se le presentaban en problemas numéricos de lápiz y papel, las variables con valores estipulados, como datos numéricos o, como signos en ecuaciones, introduciendo una idea de variable como incógnita especifica; el grupo experimental se involucraba en la elaboración de modelos experimentales, en los que debía identificar las magnitudes que varían, establecer y manipular los factores, $y$, proponer relaciones funcionales cualitativas entre éstos, asimilando una concepción de variable como magnitud que pueden relacionarse de forma funcional con otra, y cuya relación puede originar la construcción de modelos matemáticos (Morales y Díaz, 2003). 


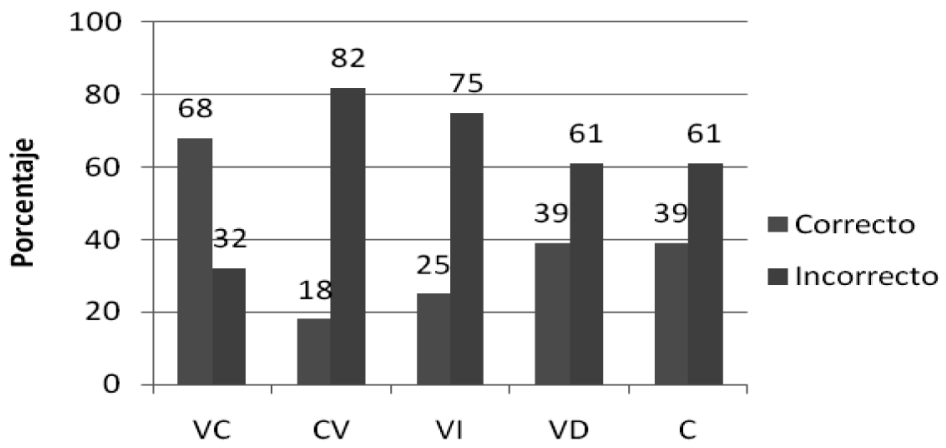

Gráfico 1. Distribución porcentual del grupo de estudiantes control de acuerdo con el uso de conceptos básicos sobre la naturaleza de las ciencias

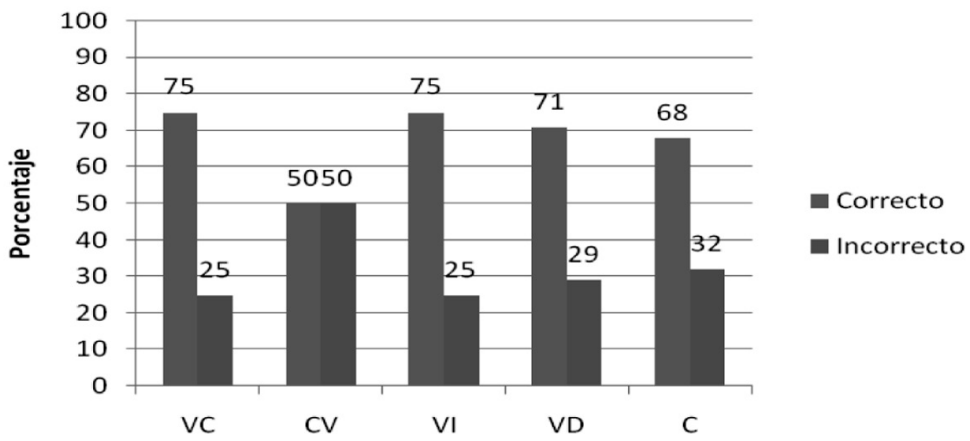

Gráfico 2. Distribución porcentual del grupo de estudiantes experimental de acuerdo con el uso de conceptos básicos sobre naturaleza de las variables. 
- Naturaleza de la variable independiente (VI)

Los resultados indican que el $25 \%$ del grupo control identifica adecuadamente a la variable independiente, mientras el $75 \%$ de este grupo no lo hace (gráfico 1). Así mismo, muestran que el $75 \%$ grupo experimental comprende lo es una variable independiente y el $25 \%$ de dicho grupo no lo comprende (gráfico 2). Las diferencias entre los dos grupos pueden deberse a que en la resolución de problemas cuantitativos realizada por el grupo control en la fase de aplicación de la estrategia didáctica tradicional, además de concebirse a las variables como números o signos que son parte de una ecuación, éstas son manipuladas de forma mecánica, sin ser comprendidas conceptualmente ni ellas, ni las relaciones funcionales cualitativas de dependencia entre las mismas, pues en estos procesos tradicionales de resolución de problemas las variables solo se manipulan en el marco de fórmulas matemáticas; mientras que, en la resolución de problemas abiertos que fue la realizada por el grupo experimental al diseñar, representar y ejecutar experimentos a manera de reformulación experimental del problema, proponiendo las variables a observar, y manipular, si se ofrecen oportunidades para reflexionar sobre la naturaleza de las variables y sobre el tipo de relación funcional que se establece entre ellas (García, 2003).

- Naturaleza de la variable dependiente (ND)

Los resultados muestran que el $39 \%$ del grupo control tienen claro el concepto de variable dependiente, pero que, el $61 \%$ de dicho grupo no (gráfico 1). Igualmente, muestran que el $71 \%$ del grupo experimental reconoce cuando una variable es dependiente y que, el $29 \%$ de dicho grupo no lo reconoce (gráfico 2). La diferencia entre los dos grupos, puede atribuirse a que en la resolución de problemas cuantitativos por parte del grupo control se manipulan mecánicamente las variables, y sus valores son acotados por el docente, además de que lo importante en este tipo de procesos de resolución de problemas es llegar a la respuesta numérica determinada, sin ofrecer la posibilidad de establecer relaciones entre las variables ni de reflexionar sobre dichas relaciones. Mientras que por el contrario, la resolución de problemas abiertos que fue realizada por el grupo experimental, incluía el diseño de modelos experimentales en los que por su naturaleza se deben estipular las variables manipuladas (independientes) y las variables observadas (dependientes) (Rubin y Tamir, 1998).

\section{- Correlación entre variables (CV)}

Los resultados muestran que el 39\% del grupo control conoce la correlación entre variables, pero que el $61 \%$ del mismo grupo no (gráfico 1). También muestran que, el $68 \%$ del grupo experimental identifican la naturaleza de la correlación pero que el $32 \%$ de dicho grupo no lo hace (gráfico 2 ). Estos resultados pueden deberse a que la resolución de los problemas cuantitativos por parte del grupo control a partir de "ejercicios muestra" provistos por el docente es de carácter mecánico, y no les dio la oportunidad de establecer relaciones cualitativas de carácter conceptual entre las variables, ni mucho menos de diferenciar cuando este tipo de relaciones correspondía a una correlación o a una relación funcional. Por el contrario, el diseño de experimentos en el marco de la resolución de problemas abiertos por parte del grupo experimental, al incluir el análisis de los datos para establecer relaciones cuantitativas entre las variables, tal vez si le permitió los estudiantes comprender la naturaleza de una correlación y, diferenciarla de una relación funcional. Por otra parte los estudiantes del grupo control al no tener la oportunidad de diseñar los 
experimentos y tener que replicarlos desde el manual de laboratorio no tuvieron la oportunidad de poner en cuestión las relaciones propuestas por el diseño experimental prefabricado que les ofrece el manual y por ende de diferenciar entre correlaciones y realciones cusales verdaderas.

De forma global se puede observar que: un $30 \%$ más de estudiantes del grupo experimental en relación con el grupo control, comprende adecuadamente los conceptos relacionados con las variables (gráfico 3 ). Los datos también muestran que la menor diferencia entre los grupos la mostró el indicador: naturaleza de la variable cuantitativa, así mismo, que la mayor diferencia entre los dos grupos se encontró en los indicadores referidos a la naturaleza específica de la variable en el marco del diseño experimental, ya sea ésta dependiente o independiente, $y$, en la capacidad para diferenciar una correlación de una relación funcional. Igualmente, de forma global se puede observar que, el indicador "concepción general de variable" fue el que presentó el menor porcentaje de respuestas correctas en los dos grupos. Las pocas diferencias entre los grupos con respecto al indicador "concepto de variable cuantitativa" pueden deberse a que este tipo de variables se usa tanto para resolver problemas cualitativos (usados en la estrategia alternativa) como cuantitativos (usados en la estrategia tradicional).

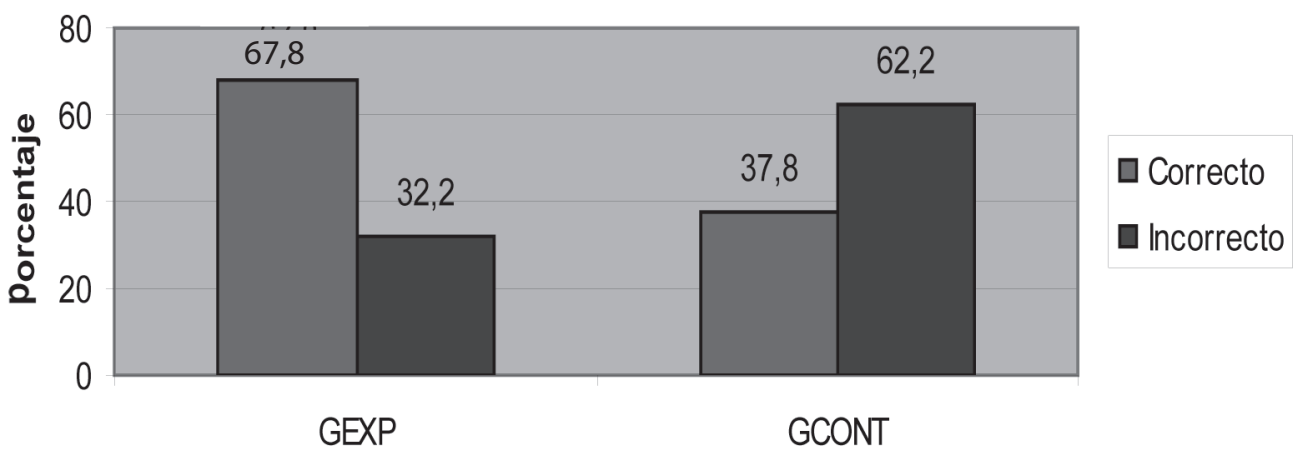

Gráfico 3. Distribución porcentual de los grupos de estudiantes experimental y control de acuerdo con el uso de conceptos básicos sobre la naturaleza de las variables.

Por otro lado, es posible, que las grandes diferencias anotadas entre los dos grupos se expliquen porque al resolver problemas cerrados en la fase de aplicación de los conocimientos, los estudiantes del grupo control usan las variables mecánicamente, realizando operaciones sin asignarles significado en la ecuación, ni construirlas con- ceptualmente, ni a ellas ni a las relaciones establecidas entre ellas, mientras que, al resolver problemas abiertos el grupo experimental, da significado a las variables y establece relaciones funcionales entre ellas, diferenciando dichas relaciones de las simples correlaciones, y dándole un uso más complejo y adecuado a las variables. Así 
mismo es importante decir, que la posibilidad de construir modelos físicos de sistemas para realizar experimentos, posibilitó a los estudiantes del grupo experimental como lo afirmaría Weisberg (2006) concentrarse en los factores primarios que explican el fenómeno a estudiar, es decir en las variables implicadas y en sus correlaciones. Estos resultados concuerdan con los de otras investigaciones que han mostrado como los estudiantes aunque realizan sin dificultad procesos algorítmicos, se les dificulta establecer el significado de los términos en una ecuación (Morales, 2003). De otro parte, el bajo porcentaje de estudiantes que mane- jan el concepto general de variable puede estar relacionado con el hecho de que en la educación tradicional por lo regular las variables son entendidas como incógnitas en una ecuación o como números, y muy pocas veces como variables verdaderas (Kuchemann, 1980).

\section{Factor conocimiento sobre el proceso de experimentación}

A continuación se presenta el análisis de los resultados en cada uno de los indicadores de este factor en ambos grupos.

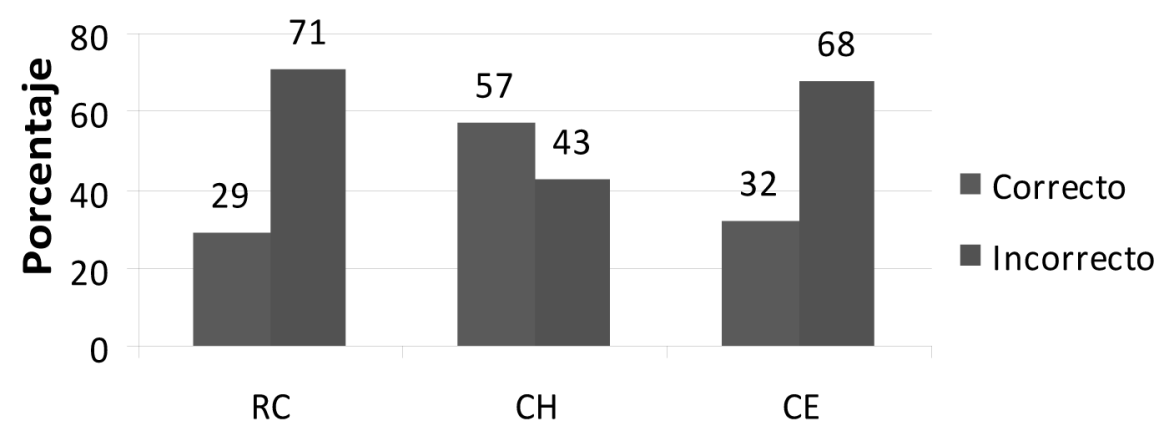

Gráfico 4. Distribución porcentual del grupo control de acuerdo con el uso de conceptos básicos sobre experimentación.

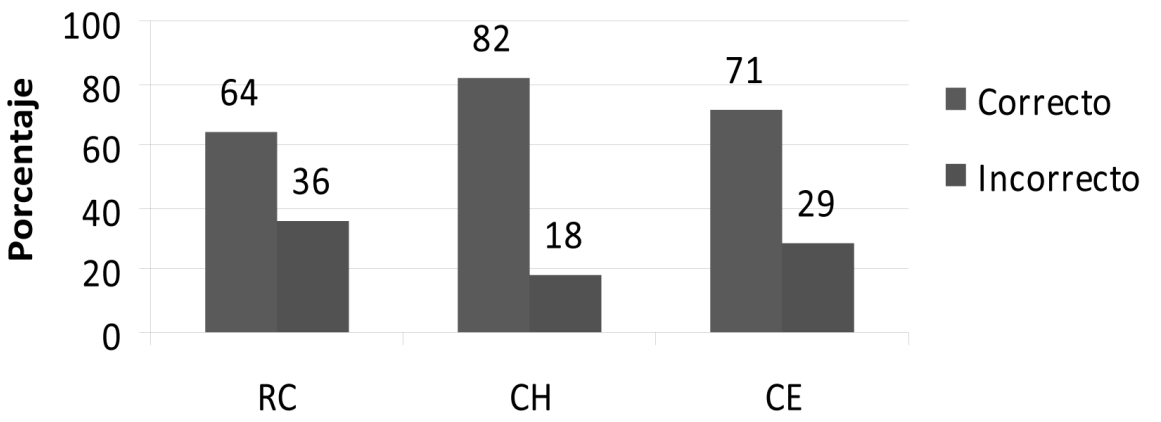

Gráfico 5. Distribución porcentual del grupo experimental de acuerdo con el uso de conceptos básicos sobre experimentación. 
- Concepto de relación causal (RC)

Los resultados indican que el $29 \%$ del grupo control sabe cuándo hay relación causal entre dos variables, pero que el $71 \%$ de dicho grupo no lo sabe (gráfico 4). También muestran como el $64 \%$ del grupo experimental determinan cuando hay relación causal entre variables, pero que el $36 \%$ de este grupo no lo hace (gráfico 5). Las diferencias entre los dos grupos pueden deberse a que al resolver los problemas algorítmicos numéricos dentro de la fase de aplicación de la estrategia tradicional, el grupo control sólo hacía uso de relaciones cuantitativas entre las variables sin identificar previamente la variable manipulada o causa y la variable observada o efecto, ni reconocer cuál de ellas ocurre primero en el tiempo, lo que sí hacía el grupo experimental al resolver problemas abiertos, diseñando y ejecutando modelos experimentales, y al analizar sus resultados para establecer o no relaciones causales entre las variables. Igualmente, es importante decir que en las prácticas experimentales prediseñadas llevadas a cabo por el grupo control los estudiantes no necesitan determinar la naturaleza explicada o causal de las variables pues esto se supone que ya está implícito en el experimento propuesto en el manual, lo que puede explicar su menor habilidad para identificar y diferenciar las relaciones de tipo causal con respecto al grupo experimental.

\section{- Concepto de hipótesis $(\mathrm{CH})$}

Los resultados muestran que el $57 \%$ del grupo control sabe qué es una hipótesis, mientras que el $43 \%$ de dicho grupo no lo sabe (gráfico 4). Así mismo, muestran como el $82 \%$ del grupo experimental, comprende el concepto de hipótesis, pero el $18 \%$ de este grupo no lo comprende (gráfico 5). Las diferencias entre los dos grupos con respecto a este indicador pueden deberse a que la resolución de problemas cerrados de aplicación realizados por el grupo control no ofrece oportunidades para construir hipótesis, por considerarlo innecesario ya que solo ajustándose al algoritmo se garantiza la resolución del problema, mientras que, al resolver problemas abiertos, el grupo experimental si tiene la oportunidad de emitir hipótesis para dirigir el diseño de modelos experimentales. Por otra parte, es importante anotar que la simple contextualización y presentación de los nuevos conceptos científicos en una estructura conceptual para acometer luego su aplicación mediante ejercicios de lápiz y papel y la realización de prácticas experimentales prediseñadas, como lo propone al estrategia tradicional utilizada con el grupo control, excluye el procedimiento de emisión de hipótesis acerca de los fenómenos pues presenta al conocimiento como algo acabado y con un carácter acumulativo, que no da lugar a dudas y que por lo tanto desecha cualquier perspectiva que considera al conocimiento como algo hipotético y en constante construcción.

\section{- Características de un experimento (CE)}

Los resultados indican que el $32 \%$ del grupo control identifica las características que debe tener un experimento, para ser confiable, y que el $68 \%$ de dicho grupo no lo hace (gráfico 4). Así mismo, muestran que el $71 \%$ del grupo experimental tiene conocimiento sobre las características que debe tener un experimento para ser confiable, pero que, el $29 \%$ de este mismo grupo no lo tiene (gráfico 5). Las diferencias entre los grupos pueden originarse en la irrelevancia de la experimentación en la resolución de problemas cuantitativos abordados por grupo control, y por su relevancia en cambio para resolver los problemas prácticos y cualitativos enfrentados por el grupo experimental (García, 2003). Estos resultados también 
pueden deberse a que en el grupo control la estrategia tradicional utilizada solo contempla como uno de sus elementos la realización de prácticas experimentales prediseñadas, en cambio en la estrategia tradicional este tipo de procedimientos son el fundamento de la misma, lo que familiariza mucho más a los estudiantes del grupo experimental con las características propias de los procedimientos expe- rimentales. Así mismo, la oportunidad de evaluar y de seleccionar sus propios diseños experimentales, propició a los estudiantes del grupo experimental oportunidades para establecer cuales eran las posibles variables extrañas que podrían alterar los resultados experimentales y así mismo de contemplar diversas formas de controlarlas, oportunidad que no tuvieron los estudiantes del grupo control.

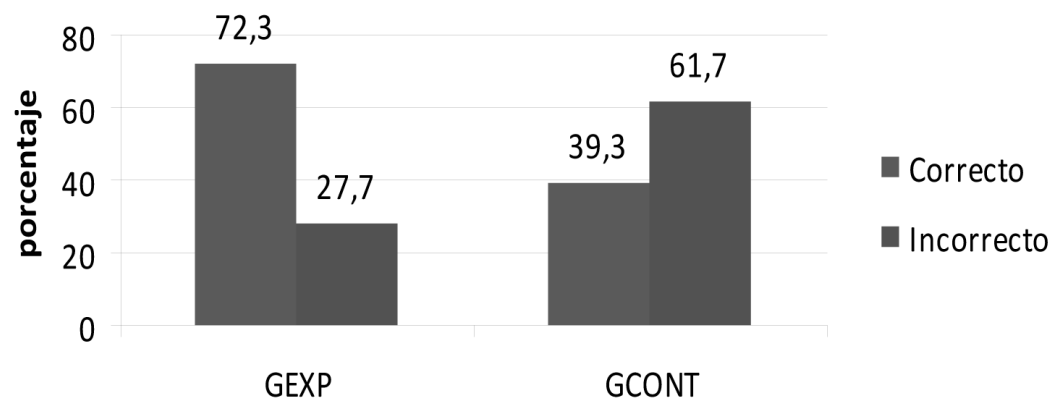

Gráfico 6. Distribución porcentual de los grupos experimental y controlde acuerdo con el uso de conceptos básicos sobre experimentación.

Un análisis global permite inferir que en promedio el $39,3 \%$ del grupo control posee conocimientos sobre conceptos básicos acerca la naturaleza de los experimentos, pero que el $61,7 \%$ de dicho grupo no los posee (gráfico 6). Además, permite observar que en promedio el $72,3 \%$ del grupo experimental tiene dichos conocimientos (gráfico 6), y que el 27,7\% no los tiene. Estas diferencias entre los dos grupos pueden deberse, tal como ya se anotó, a la resolución de problemas cerrados al contrario que la de los problemas abiertos, no requiere de la realización de actividades experimentales, y por lo tanto, del uso intencionado de conceptos como el de relación causal o el de hipótesis, y mucho menos, del conocimiento de las características de un experimento, del control de variables y de la manipulación de las mismas. Estos resultados concuerdan con otros que muestran como la construcción de modelos complementarios sobre fenómenos físicos mejora el grado de conceptualización de los estudiantes en el campo de la física y su comprensión acerca de los procesos implicados en la investigación científica (López, 2005, López, Veit y Solano, 2011).

\section{Conclusiones y recomendaciones didácticas}

Los resultados de este estudio, permiten afirmar que es favorable utilizar estrategias didácticas que involucren la resolución de problemas prácticos y abiertos al igual que 
los procedimientos de modelización experimental, cuando se espera desarrollar las habilidades de experimentación como las referidas al conocimiento de la naturaleza de las variables y de los procesos de experimentación. Desde esta perspectiva la aplicación de estrategias didácticas como la que se propone en este estudio forma al estudiante en los procesos propios de la producción de la ciencia.

Por otra parte, en este trabajo queda evidenciado que la resolución de problemas abiertos, contextualizados y de carácter práctico en el aula de ciencias experimentales, posibilita la construcción de conceptos esenciales para la comprensión de los procesos de producción científica como los de variable y de experimentación, a través del uso de procesos como la emisión de hipótesis, la elaboración de gráficas, el uso de modelos verbales de relación, el establecimiento de relaciones cualitativas y no solo cuantitativas y de, la relevancia que otorga a los procesos mismos de diseño y prueba experimental. Es por esto que se recomienda, cambiar los procesos de resolución de problemas cerrados y las prácticas experimentales tipo receta, por la integración de procesos de resolución de problemas abiertos con la realización de un trabajo experimental apoyado en los procesos de modelización, para mejorar los resultados de la labor docente en el campo de las ciencias experimentales.

Finalmente, es importante decir que la propuesta para la enseñanza de las ciencias de resolver situaciones problema utilizando los procedimientos propios de la modelización de experimentos, como fundamento, concibe a la ciencia como una actividad en la cual los sujetos se enfrentan a los fenómenos usando sus conceptos y teorías, al igual que una serie de procedimientos, con el fin de construir explicaciones descripciones y predicciones acerca de dichos fenómenos, es decir de construir nuevos conocimientos científicos. Mientras que por el contrario, la estrategia tradicional de enseñanza de las ciencias basada en la introducción expositiva de los conceptos científicos y en su posterior aplicación y demostración, parte de una concepción epistemológica absolutista desde la cual la ciencia es vista como un producto para ser consumido y no como una actividad para ser realizada, concepción epistemológica que impide pensar en la posibilidad misma del convertir a los estudiantes en futuros científicos que producen nuevos conocimientos.

\section{Bibliografía}

Arcà, M., Guidoni, P. (1989). Modelos infantiles y modelos científicos sobre la morfología de los seres vivos. Enseñanza de las Ciencias. Vol. 7, num 2, pp.162 - 167.

Black, M. (1962). Models and Metaphors: Studies in Language and Philosophy, Cornell University Press, New York

Crawford, B. A., Cullin, M. J. (2004). Supporting prospective teachers' conceptions of modelling in science. International Journal of Science Education. Vol.26, num 11, pp. 1379-1401.

Estanya, J. L. (2001). Modelización del cambio químico: hacer, pensar y escribir. Enseñanza de las Ciencias, Número extra, VI Congreso.

Fourez, G. (1994). El método científico, creación y rechazo de modelos. La construcción de conocimiento científico. Editorial Narcea. Madrid, España.

Galagovsky, L., Adúriz-Bravo, A. (2001). Modelos y analogías en la enseñanza de las ciencias naturales, el concepto de modelo didáctico analógico. Enseñanza de las Ciencias, vol 19, num 2, pp. 231-242.

García, J. J. (2003). Didáctica de las Ciencias Resolución de Problemas y Desarrollo de la Creatividad. Editorial: Magisterio. Bogotá Colombia. 
García, J. J. (2011). Didáctica de las ciencias, modelizar y resolver problemas en ciencias experimentales. Edita: Grupo Unipluriversidad. Universidad de Antioquia. Medellín. Colombia

Gilbert. (1993). Models and Modelling in Science Education. Hatfield, Herts: Association for Science Education.

Halloun, I. (1996). Schematic Modeling for Meaningful Learning of Physics. En: Journal of Research Learning of Physics. Vol 9, num 33, pp. 1019-1041.

Hestenes, D. (1992). Modeling games in the Newtonian World, American Journal Physics. 60, 732-748.

Hodson, D. (2003). Time for action: Science education for an alternative future. International Journal of Science Education. Volume 25, Issue 6.

Hogan, T. (2004). Pruebas psicológicas: una introducción práctica. Ed. Manual Moderno. México. D. F.

Ingham., Gilbert. (1991). The of analogue models by students of chemistry at higher education level. International Journal of Science Education. 22, pp. 1011-1026.

Jackson, S., Stratford, S., Kracjik, J., Soloway, E. (1995, March). Model-It: A case study of learner-centered software for supporting model building. Paper presented at the Working Conference on Technology Applications in the Science Classroom, Columbus, $\mathrm{OH}$.

Jiménez, J. D., Perales, F.J. (2002). Modèlisation et réprentation graphique de concepts. Buletin de I"union des physiciens. Vol 96 Février.

Justi, R. S. (2002). Science teachers' knowledge about and attitudes towards the use of models and modelling in learning science. International Journal of Science Education. Vol 12, num 24, pp. 1273-1292.

Küchemann, D. E. (1980). The understanding of generalised arithmetic by secondary school children. Unpublished doctoral dissertation. Chelsea College, University of London.

López, S. (2005). Aprendizaje Del concepto físico fuerza de fricción a partir de una actividad soportada en el uso del computador. Trabajo de grado maestría en Educación con énfasis en Docencia de las Ciencias Experimentales. Facultad de Educación. Universidad de Antioquia. Medellín. Colombia.

López, S; Veit, E. A; Solano, I. (2011). Modelación computacional apoyada en el uso del diagrama V de Gowin para el aprendizaje de conceptos de dinámica newtoniana. Revista Electrónica de Enseñanza de las Ciencias Vol 10, № 1, 202-226

Martinand, J.L. (1986). Enseñanza y aprendizaje de la modelización. Enseñanza de las ciencias, vol 4, num 1, pp. $45-50$.

Morales, L., y Díaz, J. (2003). El concepto de variable: dificultades de su uso a nivel universitario. Obtenido, de http://semana. mat.uson.mx

Raviolo, A., Matínez., Aznar, M. M. (2001). Desarrollo de una propuesta didáctica sobre el equilibrio químico basada en el aprendizaje de modelos. Enseñanza de las Ciencias. Número extra, VI Congreso.

Rubin, A. y Tamir, P. (1988). Meaningful learning in the school laboratory. American Biology Teacher, 50, pp.91-96.

Rubinstein, M. (1996). Patterns of problema solving. Prentice Hall. New Jersey USA.

Tomasi, J. (1999). Towards "chemical congruence" of the models in theoretical chemistry. International Journal for Philosophy of Chemistry, vol 2, num 5, pp. 79-115.

Van Driel, J. H., Verloop, N. (1999). Teachers' knowledge of models and modelling in science. International Journal of Science Education. Vol 11, num 21, pp. 1141-1153.

Weisberg, M. (2006). Three kinds of idealization, Univrsity of Pensilvania. 


\section{Anexo 1}

Prueba sobre la compresión de la naturaleza de las variables y de la experimentación

1. A continuación encontrarás enunciados que incluyen un grupo de variables, señala el enunciado que incluya únicamente variables cuantitativas:
a. Tiempo de respuesta, Velocidad, Temperatura.
b. Densidad, Masa, Tamaño (grande, mediano, pequeño, mínimo).
c. Color de pelo, Nivel socioeconómico, Concentración de alcohol en sangre.
d. Altura, edad en años, orientación sexual.

2. En la expresión "P= h.d.g" ( $P=$ Presión $\mathrm{h}=$ altura $\mathrm{d}=$ densidad, $\mathrm{g}=$ gravedad) sólo pueden ser catalogados como variables
a. Cada uno de los símbolos algebraicos usados en la expresión formal (ecuación).
b. Los valores de cada factor representado por letras en la ecuación.
c. La altura "h" y la presión "P" representadas en la ecuación.
d. La presión "P", la altura "h", la densidad, "d" y la gravedad "g".
e. La presión "P", la altura "h", y la densidad "d".

3. De acuerdo con la información proporcionada por la gráfica, selecciona la afirmación adecuada sobre la naturaleza de la variable independiente
a. El tiempo es en este caso la variable independiente.
b. El cambio en la masa es la variable independiente.
c. Cualquiera de las variables puede ser la independiente.
d. Ni el tiempo ni la masa son variables independientes. 
4. Selecciona la afirmación más adecuada acerca de la naturaleza de la variable dependiente

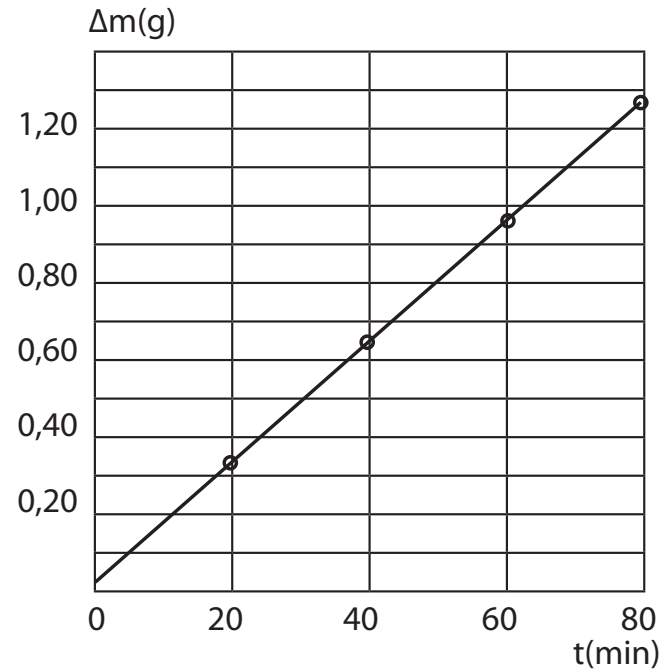

a. La variable dependiente es una propiedad de un fenómeno con capacidad para influir, incidir o afectar de una manera considerable y significativa a la variable independiente.

b. La variable dependiente es un objeto, proceso o característica a estudiar y que modifica su estado con la modificación de la variable independiente, por lo que se le considera un efecto.

c. Las variables dependientes son las que median entre las independientes (causas) y los efectos (criterios) y permiten establecer indicadores para medir la variable independiente.

d. La variable dependiente es un objeto, proceso o característica a estudiar, que modifica el estado o los valores tomados por la variable independiente, por lo que se le considera una causa.

5. Señala el caso en el cual las dos variables se encuentren correlacionadas:

a. Cuando llueve algunas veces es suspendida la energía eléctrica.

b. El consumir cigarrillo es común en enfermos de cáncer pulmonar.

c. El enojo en las chicas es causa de la poca atención de los chicos.

d. Las muchachas altas normalmente eran las rubias y europeas. 
6. Cuando afirmamos que la extrema pobreza es una de las causas de la violencia en un país, podríamos inferir que:
a. Primero se genera la pobreza y después ésta provoca la violencia.
b. La violencia genera la pobreza que sufren luego las comunidades.
c. La violencia y la pobreza son connaturales en nuestras comunidades.
d. La violencia y la pobreza se generan de una forma simultánea.

7. Una hipótesis puede ser definida como:
a. La explicación provisional de los hechos, que puede ser verdadera o falsa.
b. La explicación de los hechos que debe ser bajo toda circunstancia verdadera.
c. Una explicación de los hechos que debe ser falsa en principio para dudar de ella.
d. Explicación provisional de los hechos, por lo que es verdadera y falsa al tiempo.

8. Un experimento es confiable cuando:

a. Al manipular la variable independiente hay cambios en la dependiente.

b. Se controlan las variables extrañas que pueden afectar la variable dependiente.

c. Se utilizan materiales de laboratorio sofisticados para su realización.

d. Éste se lleva a cabo como mínimo en tres ocasiones sucesivas en el tiempo. 


\section{Anexo 2}

\section{Algunos problemas resueltos usando la modelización experimental}

\section{Cómo ahorramos energía usando ollas a presión}

¿El uso de ollas a presión permite el ahorro de energía en la cocción de los alimentos? En la actualidad por los medios de comunicación se habla de la crisis energética, debido a que las fuentes de energías como el gas, petróleo y carbón se están agotando. María es una chef que está interesada en el ahorro de energía. Atendiendo a sus deseos, María utiliza ollas a presión para cocinar los alimentos. ¿La utilización de las ollas a presión permite el ahorro de energía? ¿Cómo varía la temperatura que alcanza un cuerpo de acuerdo con la variación de la presión? ¿Cómo varía la temperatura de ebullición del agua de acuerdo con la variación de la presión atmosférica? ¿Cocinan más rápido los alimentos que están en ollas que hierven más rápido? ¿En qué lugares de Colombia se puede ahorrar más energía: en el cocinado de los alimentos de acuerdo a la presión atmosférica del lugar? Diseña y elabora un experimento que te permita establecer la relación que hay entre la temperatura, presión y volumen.

\section{¿De qué variables depende el descongelamiento de los glaciales?}

El glacial Perito Moreno está ubicado en Argentina. Dicho glacial es utilizado para abastecer de agua potable a gran parte de la población. Debido al cambio climático, el glacial Perito Moreno se está descongelando. ¿Cómo afecta el descongelamiento de los glaciales la vida humana? Si: el glacial Perito Moreno estuviera compuesto por los picos A y B de diferente masa, los dos picos tuvieran aislados térmicamente, y los dos picos recibieran la misma cantidad de rayos solares, ¿qué pico se descongelaría más rápido? ¿Cómo es la temperatura del pico A con relación al pico B durante el proceso descongelamiento? ¿Cómo es el cambio de energía interna del pico A con relación al pico B durante el proceso de descongelamiento? ¿Podemos afirmar que el pico A del glacial tiene mayor cantidad de calor que el pico B del glacial? Explica la respuesta. Tomando como referencia el glacial y el medio ambiente, ¿en el proceso de descongelamiento, ¿cuál es la dirección en la que fluye el calor? Para resolver el problema diseña y realiza un experimento que te permita extraer conclusiones sobre cómo varía la temperatura y la energía interna de un cuerpo de acuerdo con la cantidad de masa, la cantidad de calor transferido y a la temperatura inicial del cuerpo.

\section{¿Cómo influye el calor específico de la superficie de la tierra en la formación de las corrientes de aire en las zonas costeras?}

Carolina vive en Nuquí (Departamento del Chocó, Colombia) a orillas del océano Pacífico. Carolina desea aprovechar las corrientes de aire para generar energía eléctrica y abastecer las necesidades de su vivienda. ¿Qué características deben tener las superficies terrestres para que se produzcan las corrientes de aire? Teniendo como puntos de referencia las costas y el mar, ¿en qué sentido sopla el viento en las noches, en qué sentido sopla el viento en el día, por qué lo hace de esta manera? Durante un día de sol, ¿cómo crees es la temperatura de la arena en relación con el agua durante el día, cómo crees es la temperatura de la arena con relación al agua en la noche? Diseña un modelo experimental en el que se puedan generar corrientes de aire haciendo uso del calor específico de los materiales. 